University of Wollongong

Research Online

Australian Institute for Innovative Materials -

Papers

Australian Institute for Innovative Materials

$1-1-2014$

Power-law relationship between critical current density, microstructure, and the $\mathrm{n}$-value in $\mathrm{MgB2}$ superconductor wires

\author{
Ashkan Motaman \\ University of Wollongong, am107@uowmail.edu.au \\ Shaon Barua \\ University of Wollongong, sb201@uowmail.edu.au \\ Dipak Patel \\ University of Wollongong, djp485@uowmail.edu.au \\ Minoru Maeda \\ Nihon University \\ Kookchae Cheong \\ Korea Institute of Materials Science
}

See next page for additional authors

Follow this and additional works at: https://ro.uow.edu.au/aiimpapers

Part of the Engineering Commons, and the Physical Sciences and Mathematics Commons

Research Online is the open access institutional repository for the University of Wollongong. For further information contact the UOW Library: research-pubs@uow.edu.au 


\title{
Power-law relationship between critical current density, microstructure, and the n- value in MgB2 superconductor wires
}

\author{
Abstract \\ Dissipation-free MgB2 superconducting wires are valuable in terms of practical applications. Herein, we \\ have found a strong correlation between critical current density $(\mathrm{J} \mathrm{c})$ and the n-value extracted from the \\ electric field versus current density characteristic. The power-law relationship $(\mathrm{m})$ between the $\mathrm{J} c$ and \\ the $n$-value, $n \propto J m c$, represents a critical index which is strongly dependent on operating temperatures.

\section{Keywords} \\ relationship, law, value, power, critical, between, current, mgb2, density, wires, superconductor, \\ microstructure, $n$ \\ Disciplines \\ Engineering | Physical Sciences and Mathematics

\section{Publication Details} \\ Motaman, A., Barua, S., Patel, D., Maeda, M., Cheong, K., Kim, J., Dou, S. Xue. \& Al Hossain, M. Shahriar. \\ (2014). Power-law relationship between critical current density, microstructure, and the n-value in MgB2 \\ superconductor wires. Journal of Superconductivity and Novel Magnetism, 27 (7), 1643-1645.

\section{Authors} \\ Ashkan Motaman, Shaon Barua, Dipak Patel, Minoru Maeda, Kookchae Cheong, Jung Ho Kim, S X. Dou, \\ and Md Shahriar Al Hossain
}




\title{
Power-law relationship between critical current density, microstructure, and the $n$-value in $\mathrm{MgB}_{2}$ superconductor wires
}

Ashkan Motaman, Shaon Barua, Dipak Patel, Minoru Maeda ${ }^{1}$, Kookchae Cheong ${ }^{2}$, Jung Ho Kim, Shi Xue Dou, Md. Shahriar Al Hossain ${ }^{*}$

Institute for Superconducting and Electronic Materials, University of Wollongong, New South Wales 2500, Australia

${ }^{1}$ Department of Physics, College of Science and Technology, Nihon University, Tokyo 101-8308, Japan

${ }^{2}$ Nano Functional Materials Group, Korea Institute of Materials Science, Gyeongnam 642-831, Republic of Korea

\begin{abstract}
Dissipation-free $\mathrm{MgB}_{2}$ superconducting wires are valuable in terms of practical applications. Herein, we have found a strong correlation between critical current density $\left(J_{c}\right)$ and the $n$-value extracted from the electric field versus current density characteristic. The power-law relationship ( $m$ ) between the $J_{\mathrm{c}}$ and the $n$-value, $n \propto J_{\mathrm{c}}{ }^{m}$, represents a critical index which is strongly dependent on operating temperatures.
\end{abstract}

Keywords: magnesium diboride, critical current density, power law, $n$-value

*Corresponding author: Tel. + 6124221 3384; Fax. 6124221 5731; Email: shahriar@uow.edu.au 
Very recently, further enhancement of the critical current density, $J_{\mathrm{c}}$, and the irreversibility field, $B_{\mathrm{irr}}$, has been reported for carbon doped $\mathrm{MgB}_{2}$ wires with malic acid additive, produced by the chemical solution method [1-5]. In terms of monofilament conductor, a high $J_{\mathrm{c}}$ of 23,000 $\mathrm{Acm}^{-2}$ at $4.2 \mathrm{~K}$ and $10 \mathrm{~T}$ was achieved in our previous study [6]. However, an inherent problem for $\mathrm{MgB}_{2}$ wire is still the low mass and low volume densities, which are due to its porous nature. In addition, commonly used carbon dopant, which is known to be effective for achieving a larger upper critical field, tends to aggregate at grain boundaries as a residue, due to the limit of carbon solubility in the $\mathrm{MgB}_{2}$ structure. Thus, both porosities and residual carbon could act as major current-limiting factors, which are directly reflected in the electric field $(E)$ - current density $(J)$ characteristic.

The E-J characteristic is commonly known to be a crucial index for optimization of the design of superconducting applications. For example, typical values of $n$ for "persistent magnet-grade" conductors are required to be 50-100. However, the index resistance due to low $n$-value, $10-20$, of $\mathrm{MgB}_{2}$ at higher operating temperature close to $25 \mathrm{~K}$ and 3-4 T applied magnetic field is expected to make it impossible to operate an $\mathrm{MgB}_{2}$-based magnet in persistent mode, i.e., $<0.01 \mathrm{ppm} / \mathrm{hr}$, even if all joints are superconducting [7]. From the viewpoint of materials, a high quality sample usually shows a larger $n$ value, defined by the close approximation of the $E-J$ characteristic by a curve $E \propto J^{n}$. This means more homogeneous microstructure causes larger $n$-value. Compared with high temperature superconductors [8], $\mathrm{MgB}_{2}$ shows a sharper transition from the superconducting state to the normal state with increasing transport current. However, its voltage rise due to its low $n$-value cannot be negligible, even below the critical current, because it can lead to electrical and thermal dissipation. Thus, dissipation-free superconductor wires have been the primary issue for practical application. For these reasons, it is necessary to investigate the effects of carbon dopant on the $n$-value and microstructure. In this study, we have evaluated in detail the relationship between $J_{\mathrm{c}}$, microstructure, and the $n$-value in the best performance carbon doped wire obtained through malic acid doping.

$\mathrm{MgB}_{2} / \mathrm{Nb} /$ Monel monofilament wire with $10 \mathrm{wt} \%$ malic acid additive and un-doped $\mathrm{MgB}_{2}$ wire as a reference for comparison, both fabricated by Hyper Tech Research Inc., were studied in this work. Details of the experiments have been described elsewhere $[1,6]$. Transport critical current up to 400 A was measured by using the standard four-probe method with a criterion of $1 \mu \mathrm{Vcm}^{-1}$. The magnetic field was increased up to $18 \mathrm{~T}$, while the temperature was varied within the range of $4.2 \mathrm{~K}$ to $30 \mathrm{~K}$. The $n$-values were determined from the slope in the plot of $\log E$ versus $\log J$ in the electric field $(E)$ range from 0.1 to $10 \mu \mathrm{Vcm}^{-1}$, based on the power law, $E_{c}=E\left(V / V_{c}\right)^{n}$, Where the $E_{\mathrm{c}}$ represents the critical potential $V_{\mathrm{c}}$ in unit length. The critical temperature ( $T_{\mathrm{c}}$ ) was determined by AC susceptibility measurements at $f=76.97 \mathrm{~Hz}$ with $\mu_{0} H_{\mathrm{c}}=50$ 
$\mu T$, where $H_{\mathrm{c}}$ is the upper critical field. Microscopic studies were carried out using a JEOL JEM-2500SES, a Cs-corrected STEM equipped with a Gantan 776 EELS (Enfina 1000).

In order to determine the optimal sintering temperature, transport $J_{\mathrm{C}}$ values for malic acid doped wires sintered at different temperatures were collected and are shown in Figure 1. Quite interestingly, even when sintering at a temperature as low as $550^{\circ} \mathrm{C}$, the $J_{\mathrm{c}}$ is still as high as $\sim 18,000 \mathrm{Acm}^{-2}$ at $4.2 \mathrm{~K}$ and $10 \mathrm{~T}$. Further enhancement of the $J_{\mathrm{c}}$ is achievable by slightly increasing the sintering temperature and decreasing the sintering time, for example, $J_{\mathrm{c}}$ was $25,000 \mathrm{Acm}^{-2}$ when the sample was sintered at $600^{\circ} \mathrm{C}$ for 4 hours. At an external magnetic field of $11.7 \mathrm{~T}$, the $J_{\mathrm{c}}$ is still around $10,000 \mathrm{Acm}^{-2}$. On the other hand, the $J_{\mathrm{c}}$ of the reference un-doped wire sintered at $650^{\circ} \mathrm{C}$ for $30 \mathrm{~min}$ was about an order of magnitude lower, about 2,800 $\mathrm{Acm}^{-2}$ at $4.2 \mathrm{~K}$ and $10 \mathrm{~T}$. Interestingly, transport $J_{\mathrm{c}}$ values were found to be strongly dependent on the sintering temperature, which can lead to differences in the $T_{\mathrm{c}}$. The $T_{\mathrm{c}}$ of the un-doped wire is $36 \mathrm{~K}$, but $T_{\mathrm{c}}$ of all the carbon-doped wires is approximately $34 \mathrm{~K}$. Corresponding to this reduction in $T_{\mathrm{c}}$, the $a$-lattice parameter of the malic-acid doped wires was decreased from 3.0832(2) to 3.0758(2) Å. This can be attributed to volume shrinkage due to carbon substitution.

Among them, we choose the best performance wire sintered at $600^{\circ} \mathrm{C}$ for 4 hours and temperature dependence of $J_{\mathrm{c}}(B)$ is shown in Figure 2(a). We observed that the $J_{\mathrm{c}}$ normally decreased with increasing magnetic field. In addition, the slope of $J_{\mathrm{c}}(B)$ became steeper with increasing operating temperature. It is noteworthy that critical current density values at $4.2 \mathrm{~K}$ and $20 \mathrm{~K}$ are still above $10^{4} \mathrm{Acm}^{-2}$, even in fields up to $10 \mathrm{~T}$ and $5 \mathrm{~T}$, respectively. The corresponding $n$-values are shown in Figure 2(b). This behaviour is similar to that of $J_{\mathrm{c}}(B)$ under different operating temperatures. Here, our natural question is then why the $n$-value is crucial. It is well known that electrical and thermal properties are significantly degraded at inhomogeneous parts of a sample, thereby reducing the stability of the whole system $[7,9,10]$. In particular, the nonuniformity in the conductor can result in "hot spot formation" that leads to localized thermal quenching [11]. For $\mathrm{Nb}_{3} \mathrm{Sn}$, the $n$-value sharply decreases as the field approaches the upper critical field, and the critical current also decreases sharply [8, 12]. On the other hand, for $\mathrm{YBa}_{2} \mathrm{Cu}_{3} \mathrm{O}_{7}$ (YBCO) coated conductor, the decrease in the critical current density is rather gradual, and the $n$-value also changes gradually at the same time [13]. This proves that different kinds of superconducting wires show unexpected $n$-value behaviour under magnetic fields. This is why it would be informative if we can understand behaviour of $n$-value for the $\mathrm{MgB}_{2}$ wire under different fields and operating temperatures. 
As was noted, effect of dimensional defects in $\mathrm{MgB}_{2}$ necessary to study on the current-carrying performances, i.e., carbon doping. When carbon is doped into the $\mathrm{MgB}_{2}$, it is believed that it substitutes for boron, stiffens the optical $\mathrm{E}_{2 \mathrm{~g}}$ phonon mode, which is strongly linked with anisotropic $\sigma$ bands, hence, lowers the transition temperature [14]. Even if carbon doped wire still shows better $J_{\mathrm{c}}$, inhomogeneous microstructure remains rooms for degradation of $n$-value. Figure 3 shows a lattice distortion inside $\mathrm{MgB}_{2}$ grain. Many dislocations can be occurred, resulting in severe crystallographic imperfections. These structural defects produce an increase in the impurity scattering rate, which thereby enhance the upper critical field and the high-field $J_{\text {c. }}$ However, it simultaneously leads to lower $n$-value.

The power-law relationship between the $J_{\mathrm{c}} \mathrm{s}$ and the $n$-values of wire evaluated over all temperature ranges is shown in Figure 4. The index $m$ value was estimated to be 0.399 , which is comparable to our previous result [14]. In the literatures, Martinez et al, Kitaguchi et al, and Li et al reported that $m$-indexs were estimated to be $0.72,0.69$, and 0.5 , respectively [1517]. Among these, HIPed bulk samples show larger $m$-value [16]. This means it depend on microstructure even if $m$-index does not vary much. It is noteworthy that higher $J_{\mathrm{c}}$ values can be attributed to larger $n$-values. From our results, the quantitative $n$-value of current $\mathrm{MgB}_{2}$ wire is demonstrated to need further improvement, especially for persistent magnetmode.

In summary, the correlation between $J_{\mathrm{c}}$ and the $n$-value in $\mathrm{MgB}_{2}$ superconducting wires was evaluated under different operating temperatures. From the $E-J$ characteristics, we found that $J_{\mathrm{c}}$ is proportional to the $n$-value, which is strongly dependent on the operating temperature. Large $n$-values $>30$ are expected to make it possible to operate an $\mathrm{MgB}_{2}$-based magnet in persistent mode at $20 \mathrm{~K}$.

Acknowledgements: This work was supported by the Australian Research Council (Grant No. DE130101247), a 2013 AIIM-CRG and 2014 UOW-URC grants 


\section{References}

[1] J. H. Kim, S. Zhou, M. S. A. Hossain, A. V. Pan and S. X. Dou: Appl. Phys. Lett. 89 (2006) 142505.

[2] Z. S. Gao, Y. M. Ma, X. P. Zhang, D. L. Wang, Z. G. Yu, H. Yang, H. H. Wen and E. Mossang: J. Appl. Phys. 102 (2007) 013914.

[3] B. H. Jun, Y. J. Kim, K. S. Tan and C. J. Kim: Supercond. Sci. Technol. 21 (2008) 105006.

[4] Y. J. Kim, B. H. Jun, K. S. Tan, B. G. Kim, J. M. Sohn and C. J. Kim: Physica C 468 (2008) 1372.

[5] A. Vajpayee, V. P. S. Awana, G. L. Bhalla, P. A. Bhobe, A. K. Nigam and H. Kishan: Supercond. Sci. Technol. 22 (2009) 015016.

[6] J. H. Kim, S. X. Dou, S. Oh, M. Jercinović, E. Babić, T. Nakane and H. Kumakura: J. Appl. Phys. 104 (2008) 063911.

[7] J. H. Kim, S. X. Dou, A. Matsumoto, S. Choi, T. Kiyoshi and H. Kumakura: Physica C 470 (2010) 1207-1210.

[8] A. Rimikis, R. Kimmich, Th. Schneider: IEEE Trans Appl Supercond. 10 (2000) 1239-1242.

[9] G. Z. Li, Y. Yang, M. A. Susner, M. D. Sumption and E. W. Collings: Supercond. Sci. Technol. 25 (2012) 025001.

[10] A. Stenvall, A. Korpela, R. Mikkonen and G. Grasso: Supercond. Sci. Technol. 19 (2006) 184-9

[11] P. Kovac, B. Birajdar, I. Husek, T. Holubek and O. Eib: Supercond. Sci. Technol. 21 (2008) 045011.

[12] H. Murakami, A. Ishiyama, H. Ueda, N. Koizumi, and K. Okuno: IEEE Transactions on Applied Superconductivity 17 (2007) 2.

[13] N. Amemiya, S. Kasai, K. Yoda, Z. Jiang, G. A. Levin, P. N. Barnes and C. E. Oberly: Supercond. Sci. Technol. 17 (2004) 1464-1471.

[14] J.H. Kim, S.X. Dou, A. Matsumoto, S. Choi, T. Kiyoshi, H. Kumakura: Physica C 470 (2010) 1207-1210

[15] E.Martinez, A.Angural , S.I.Schlachter and P.Kovač: Supercond. Sci. Technol.22 (2009) 015014

[16] H.Kitaguchi, A.Matsumoto, H.Hatakeyama and H.Kumakura: Physica C 401 (2004) 246

[17] G.Z.Li, Y.Yang, M.A.Susner, M.D.Sumption and E.W.Collings: Supercond. Sci. Technol. 25 (2012) 025001 (10pp)

\section{Figure Captions}

Fig. 1. Magnetic field dependence of the transport $J_{\mathrm{c}}$ for malic acid-doped $\mathrm{MgB}_{2}$ wires with different sintering temperatures.

Fig. 2. (a) Magnetic field dependence of the transport $J_{c}$, and (b) magnetic field dependence of the $n$-value at 4.2, 10, 14, 20, 25, and $30 \mathrm{~K}$ for malic acid-doped $\mathrm{MgB}_{2}$ wires.

Fig. 3. Lattice distortion inside carbon doped $\mathrm{MgB}_{2}$

Fig. 4. Correlation between the $J_{\mathrm{c}}$ and the $n$-value for malic acid-doped $\mathrm{MgB}_{2}$ wire in the operating temperature range of 4.2 to $30 \mathrm{~K}$. 


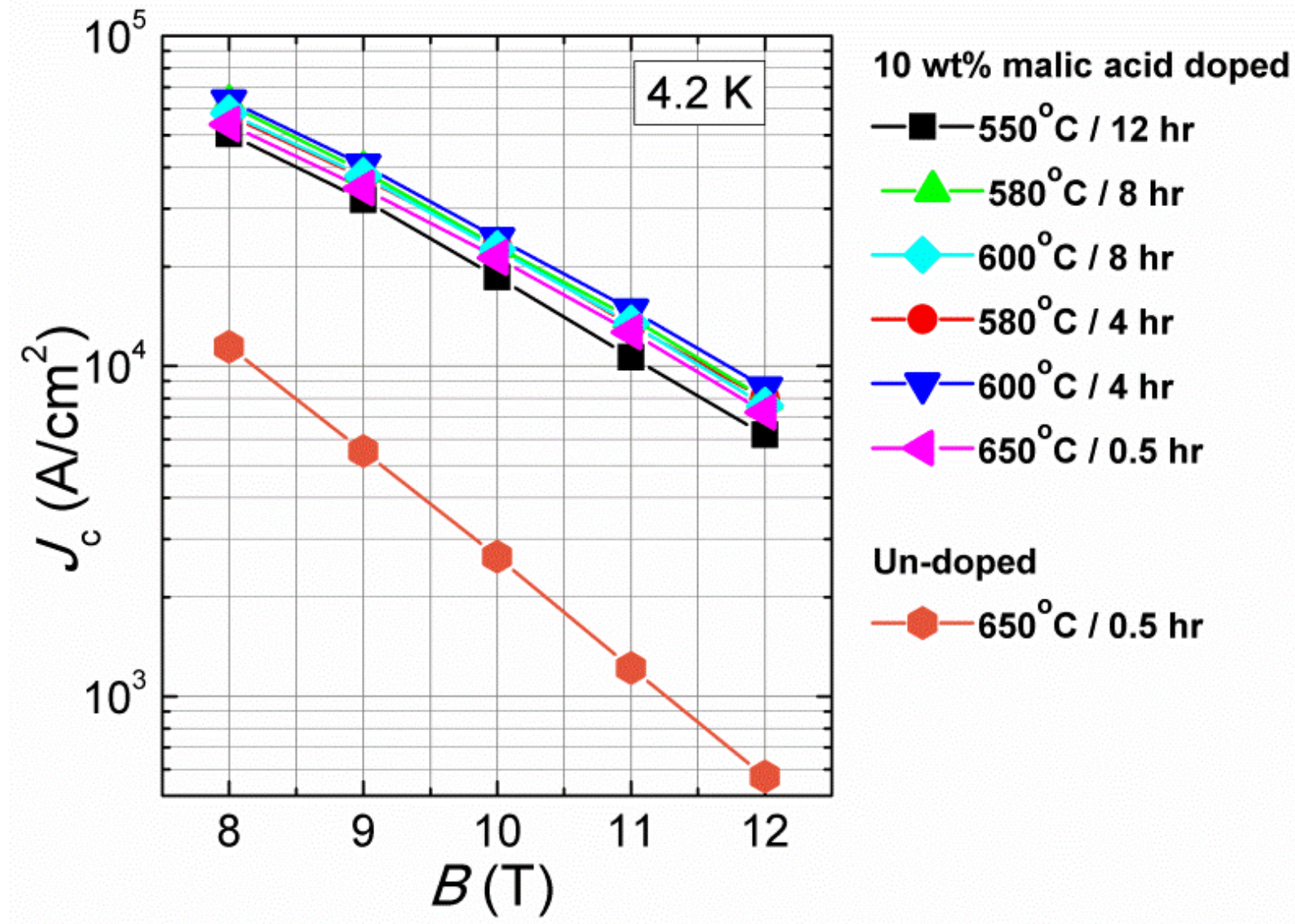

Fig. 1 


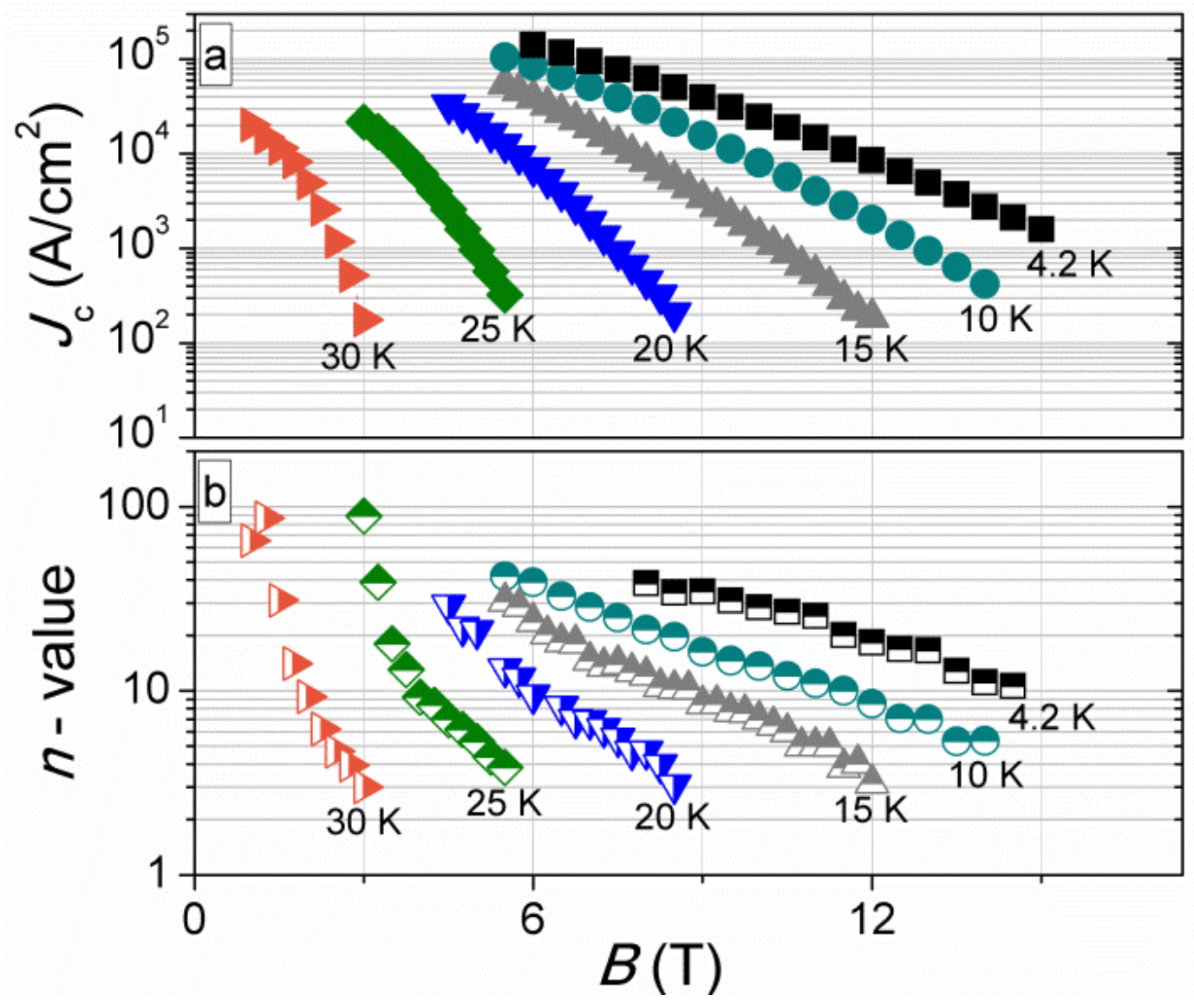

Fig. 2

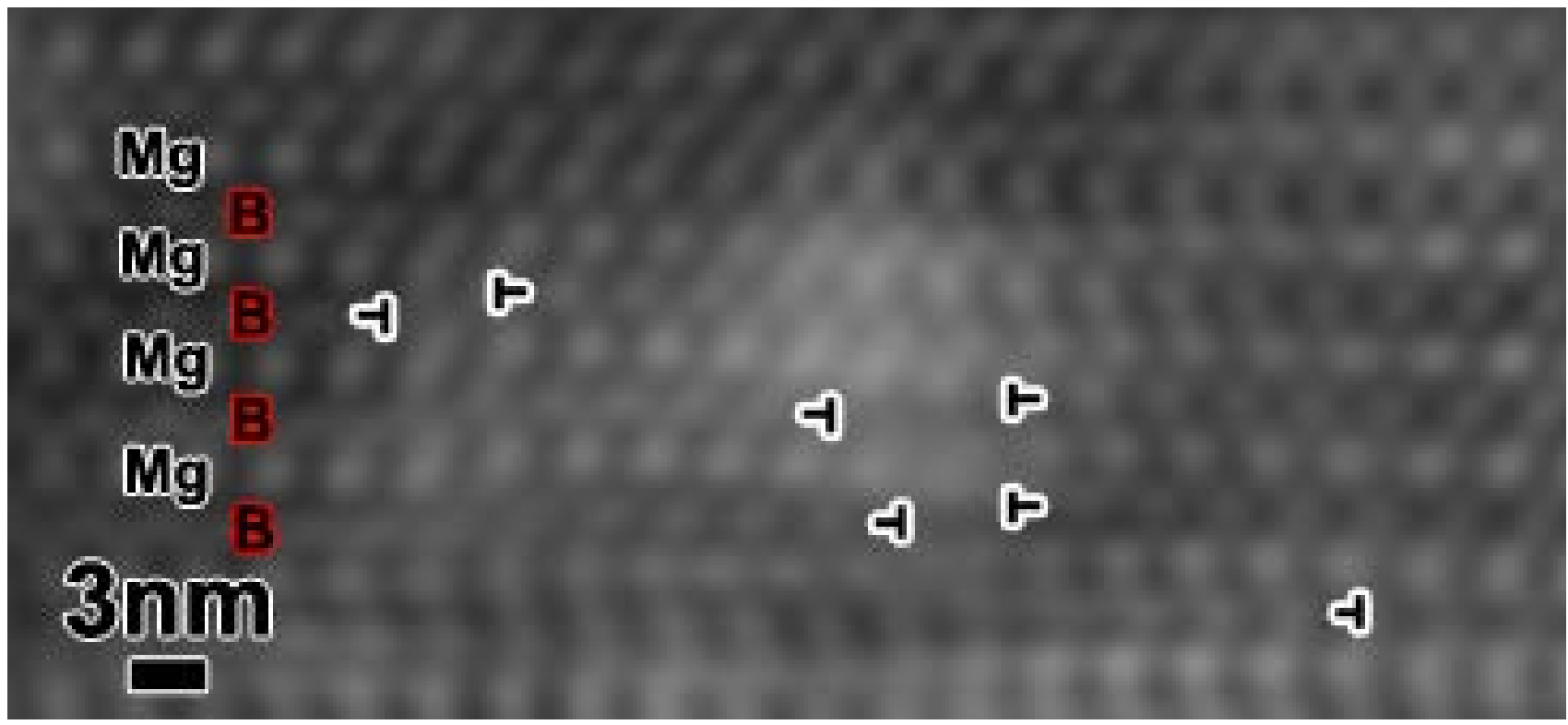

Fig. 3 


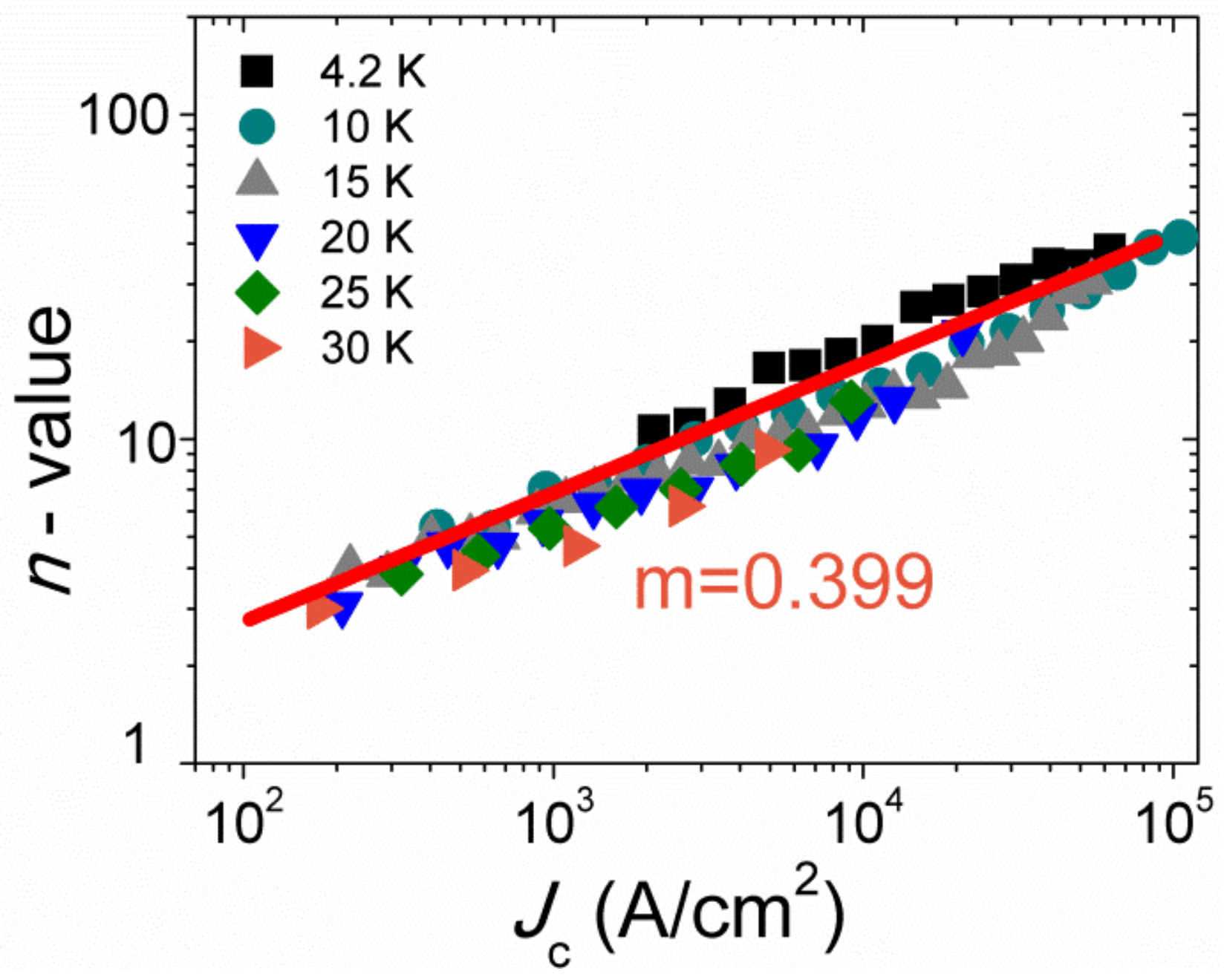

Fig. 4 